\title{
Mapeo de áreas regadas usando datos geoespaciales y teledetec- ción en el municipio de Caudete de las Fuentes (Valencia).
}

\author{
López-Pérez, Esther1', Sanchis-Ibor, C. ${ }^{2}$, Jiménez Bello, M.A. ${ }^{3}$, Rubio-Martin, A. ${ }^{1}$, Macian-Sorribes, H.1 , García-Prats,
} A. ${ }^{3}$, Manzano-Juárez, J. ${ }^{4}$, García-Mollá, M. ${ }^{5}$, Pulido-Velazquez, M. ${ }^{6}$

\footnotetext{
${ }^{1}$ Técnico Superior de Investigación en el Instituto Universitario de Investigación de Ingeniería del Agua y Medio Ambiente (IIAMA), Universitat Politècnica de València Universitat, Camí de Vera s/n, 46022 València. estloppe@upvnet.upv.es; adrumar@cam.upv.es; hecmasor@upv.es

2 Técnico Superior de Investigación en el Centro Valenciano de Estudios sobre el Riego (CVER), de la Universitat Politècnica de València, Camí de Vera, s/n 46022 València. csanchis@hma.upv.es

${ }^{3}$ Profesor Contratado Doctor, Inst. U. Ingeniería del Agua y M.A (IIAMA), Universitat Politècnica de València, Camino de Vera, s/n 46022 Valencia.mijibar@dihma.upv.es

${ }^{4}$ Profesor Contratado Doctor. Centro Valenciano de Estudios sobre el Riego (CVER), de la Universitat Politècnica de València, Camí de Vera, s/n 46022 València. juamanju@agf.upv.es

${ }^{5}$ Profesora Titular. Centro Valenciano de Estudios sobre el Riego (CVER), de la Universitat Politècnica de València, Camí de Vera, s/n 46022 València.mgarmo@upvnet.upv.es

${ }^{6}$ Catedrático y Director del Inst. U. de Investigación de Ingeniería del Agua y Medio Ambiente (IIAMA), Universitat Politècnica de València Universitat, Camí de Vera s/n, 46022 Valencia. mapuve@hma.upv.es
}

Resumen: Las políticas de control del uso agrícola de aguas subterráneas mediante la inspección de contadores se han demostrado caras y poco eficientes, mientras que en algunos estudios se ha obtenido resultados prometedores mediante la teledetección. El rápido progreso de las tecnologías de teledetección ha hecho posible su aplicación para la identificación de áreas regadas, y los nuevos sensores y técnicas de inteligencia artificial abren nuevas oportunidades a mejorar su eficacia y precisión. Nuestro trabajo propone una metodología de bajo coste y eficiente para detectar viña en riego a escala de parcela con el fin de mejorar la gestión colectiva de aguas subterráneas. A partir de información oficial se ha distinguido la superficie regada con técnicas de análisis de aprendizaje automático, empleando variables que condicionan el estado hídrico de la planta para la temporada de riego 2019. La metodología calcula la humedad del suelo con el método OPTRAM (OPtical TRApezoid Model) de análisis multitemporal de imágenes procedentes de plataformas satelitales. Estos datos son integrados en un SIG junto a información climática, topográfica e información propia del cultivo. Finalmente, en base a inventarios de verdad-terreno se aplica un clasificador de aprendizaje automático para estimar la superficie regada con agua procedente del acuífero. Los resultados obtenidos presentan una precisión general del $94.7 \%$. Su evaluación aporta un error medio cuadrático de 0.163 y R-cuadrado de 0.874 . La alta precisión y los bajos niveles de error obtenidos permiten validar la metodología empleada, que presenta potencial de mejora mediante una mayor alimentación del proceso de aprendizaje automático, que se aplicará en breve a otros cultivos leñosos.

Palabras clave: área regada; teledetección; OPTRAM; agua subterránea; inteligencia artificial; gestión colectiva 


\title{
Mapping irrigated areas using geospatial data and remote sens- ing in Caudete de las Fuentes (Valencia, Spain).
}

\author{
López-Pérez, Esther1 ${ }^{1}$, Sanchis-Ibor, C², Jiménez Bello, MA ${ }^{3}$, Rubio-Martin, Adrià1, Macian-Sorribes, H. ${ }^{1}$, García- \\ Prats, A. ${ }^{3}$, Manzano-Juárez, J. ${ }^{4}$, García-Mollá, M ${ }^{5}$, Pulido-Velázquez, M $^{6}$
}

\footnotetext{
${ }^{1}$ Técnico Superior de Investigación en el Instituto Universitario de Investigación de Ingeniería del Agua y Medio Ambiente (IIAMA), Universitat Politècnica de València Universitat, Camí de Vera s/n, 46022 València. estloppe@upvnet.upv.es; adrumar@cam.upv.es; hecmasor@upv.es

2 Técnico Superior de Investigación en el Centro Valenciano de Estudios sobre el Riego (CVER), de la Universitat Politècnica de València, Camí de Vera, s/n 46022 València. csanchis@hma.upv.es

${ }^{3}$ Profesor Contratado Doctor, Inst. U. Ingeniería del Agua y M.A (IIAMA), Universidat Politècnica de València, Camino de Vera, s/n 46022 Valencia.mijibar@dihma.upv.es

4 Profesor Contratado Doctor. Centro Valenciano de Estudios sobre el Riego (CVER), de la Universitat Politècnica de València, Camí de Vera, s/n 46022 València. juamanju@agf.upv.es

${ }^{5}$ Profesora Titular. Centro Valenciano de Estudios sobre el Riego (CVER), de la Universitat Politècnica de València, Camí de Vera, s/n 46022 València.mgarmo@upvnet.upv.es

${ }^{6}$ Catedrático y Director del Inst. U. de Investigación de Ingeniería del Agua y Medio Ambiente (IIAMA), Universitat Politècnica de València Universitat, Camí de Vera s/n, 46022 Valencia. mapuve@hma.upv.es
}

\begin{abstract}
The control of groundwater for agricultural use through water meters has proven to be expensive and inefficient in numerous cases, while some studies have shown promising results using remote sensing techniques. Recent developments of remote sensing technologies have improved the capacity to detect and quantify irrigated areas, and new sensors and machine learning techniques have opened new opportunities for increasing the precision and efficiency of these methodologies. Our study proposes a cheap and efficient methodology to detect irrigated vineyards at plot scale, in order to improve the collective management of groundwater. Irrigated areas have been detected by applying machine learning techniques using determining variables of the plant water content from the dry season of 2019. The methodology calculates the soil moisture by making use of Optical TRApezoid Model (OPTRAM), supported by multitemporal analysis of satellites images. This data has been integrated into a GIS with climatic and topographic parameters, and crop data. Finally, a machine learning classifier has been applied to estimate the extent of areas irrigated with groundwater. Results show a general accuracy of $94.7 \%$. Data evaluation provides a mean square error of 0.163 and R-square of 0.874 . The high accuracy enables the validation of the described methodology, which still has potential for improvement by enlarging the learning dataset, which will soon be applied to other wood crops.
\end{abstract}

Keywords: irrigated area, remote sensing, OPTRAM, groundwater, machine learning, collective management. 


\section{Congreso Nacional de Riegos CARTAGENA 2021}

\section{Introducción}

La expansión del riego con aguas subterráneas en regiones áridas y semiáridas conlleva con demasiada frecuencia la sobreexplotación de los acuíferos, con consecuencias negativas para la viabilidad y rentabilidad de la agricultura, para el futuro de la producción de alimentos y para la sostenibilidad de los ecosistemas acuáticos [1,2]. Ante este problema, generalizado en las cuencas mediterráneas, los expertos han propuesto varios enfoques, algunos basados en la mejora de la gobernanza, otros en medidas de control y sanciones coercitivas y, cada vez más, también se aplican políticas mixtas denominadas de 'palo y zanahoria' [3-7].

En casi todos los casos, es fundamental contar con herramientas para estimar la superficie regada, ya que, sin un flujo de información adecuado sobre la evolución del uso común del recurso, es mucho más difícil desarrollar políticas impulsadas por el Estado o poner en marcha instrumentos de gestión colectiva que permitan la explotación sostenible $[8,9]$. Las metodologías aplicadas a la identificación de áreas de cultivo y superficies de riego que utilizan datos geoespaciales y sensores de observación de la Tierra son de gran utilidad en este contexto y están comenzando a aplicarse con relativo éxito en algunas regiones del mundo [10-12].

El primer mapa digital mundial de áreas regadas basado en información cartográfica fue desarrollado en 1999 por la Organización de las Naciones Unidas para la Agricultura y la Alimentación (FAO). Desde entonces, se ha ido mejorando la metodología hasta llegar a un mapa global con datos nacionales oficiales e información georreferenciada de agricultura de regadío de 187 países organizado a través de la iniciativa AQUASTAT [13].

El rápido progreso de las tecnologías de observación remota ha hecho posible su aplicación como una nueva y esencial fuente de datos para aplicaciones ambientales y para el seguimiento de cambios en la agricultura. El diseño de un mapa de superficie regada es quizás una de las aplicaciones más buscadas de la observación remota en la gestión del agua [14-16].

La teledetección mediante métodos ópticos utiliza algoritmos matemáticos que describen la relación entre la señal capturada por el sensor y el contenido de agua en la superficie. Los métodos de clasificación de series temporales de imágenes aéreas son ampliamente utilizados para el mapeo de cultivos. Estos métodos utilizan índices de vegetación en el rango óptico de la reflectancia espectral emitida por la vegetación durante su fase de desarrollo [14, 17, 18]. Recientemente, para la estimación de las áreas regadas a escala global con teledetección, se emplea la humedad del suelo captada por el sensor, resultado de aplicar modelos de superficie terrestre [19]. Otros estudios emplean para su cálculo el 'Método del triángulo' que consiste en incorporar la temperatura infrarroja superficial TIR e índices de vegetación para obtener un índice de humedad del cultivo [20, 21].

Recientemente, se ha desarrollado un nuevo modelo que relaciona datos térmicos de reflectancia de onda corta (SWIR) y datos ópticos mediante índices de vegetación. Se basa en el método del triángulo y es conocido como OPtical TRApezoid Model (OTRAM) [22]. Los buenos resultados obtenidos a pequeña escala por este método para el cálculo de la humedad del cultivo [23-25] nos han impulsado a desarrollar un método económico y eficiente para estimar áreas regadas en viñedo a través de sensores remotos. La metodología utiliza algoritmos de aprendizaje automático empleando variables condicionantes del estado hídrico del campo y el índice de humedad del cultivo. Los resultados pueden ser utilizados para mejorar la gobernanza del agua a través de una gestión colectiva transparente.

\section{Materiales y métodos.}

La metodología desarrollada parte de la integración de información geoespacial en la zona seleccionada a través de funciones de superposición de datos climáticos, topográficos, y de morfología y estado hídrico del cultivo. Esta información es incorporada en un Sistemas de Información Geográfica (SIG) en forma de capas georreferenciadas. Posteriormente los datos son analizados junto a información 


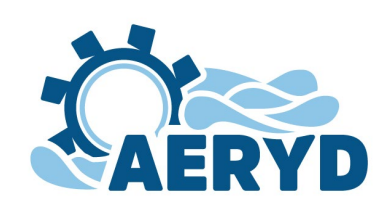

\section{Congreso Nacional de Riegos CARTAGENA 2021}

real del estado de riego de un conjunto de parcelas inventariadas mediante algoritmos de clasificación con técnicas de aprendizaje automático. Finalmente, se generan los resultados de la estimación del estado hídrico en la zona. En la presente contribución se ha empleado como área de estudio un municipio del interior de la provincia de Valencia, Caudete de las Fuentes.

\section{1. Área de estudio.}

Caudete de las Fuentes es un municipio de la comarca de Utiel-Requena (Provincia de Valencia) junto al límite entre la Comunidad Valenciana y la de Castilla-La Mancha. Este municipio se extiende sobre una superficie de $34.5 \mathrm{~km}^{2}$ de los cuales, según catastro, 2.856 ha se dedican a la producción de uva para vinificación. El clima es mediterráneo con un marcado carácter continental (Csa y Csb Köppen-Geiger). La temperatura media anual es de $14^{\circ}$ y la precipitación media anual es de $434 \mathrm{~mm}$. La parte más seca del año es de abril a septiembre y coincide con la temporada de riego, entre los meses de junio y agosto.

Los viñedos de Caudete de las Fuentes aprovechan el agua de la masa subterránea de RequenaUtiel, que está situada en la margen occidental del altiplano del mismo nombre. La masa de agua tiene una superficie de $987,9 \mathrm{~km}^{2}$ y su altitud oscila entre los 600 y los $1.200 \mathrm{~m}$ sobre el nivel del mar. En 1995, según las estadísticas de la Generalitat (VAB, 2021), la superficie regada de Requena-Utiel ocupaba $1.738 \mathrm{ha}$, de las cuales 1.279 ha eran forrajes y hortalizas de verano, y otras 449 se dedicaban al viñedo. El resto de la comarca estaba ocupada por cultivos de secano, 58.524 ha de las cuales 44.445 ha eran viñedos. Sin embargo, en 2019 la superficie regada se había ampliado significativamente hasta alcanzar las 11.660 ha de viñedos, 1.546 de almendros y 1.415 de otros cultivos, es decir se ha multiplicado por ocho en 24 años. La creación de estos nuevos sistemas de riego se llevó a cabo en la mayoría de los casos gracias a la instalación de sistemas de riego a goteo y el emparrado de la vid.

En 2016, la Confederación Hidrográfica del Júcar (CHJ) aprobó el Plan de explotación de la masa de agua subterránea Requena-Utiel, que ha establecido una dotación hídrica de $450 \mathrm{~m}$ /ha/año para cultivos leñosos para años húmedos ( $900 \mathrm{~m}^{3} / \mathrm{ha} /$ año para años secos) en la zona con concesión administrativa, [26]. Actualmente, la CHJ estima un área con derechos de agua consolidados de 19.495 ha y la administración autonómica calcula 14.621 ha de regadío (de las cuales 11.660 viñedos).

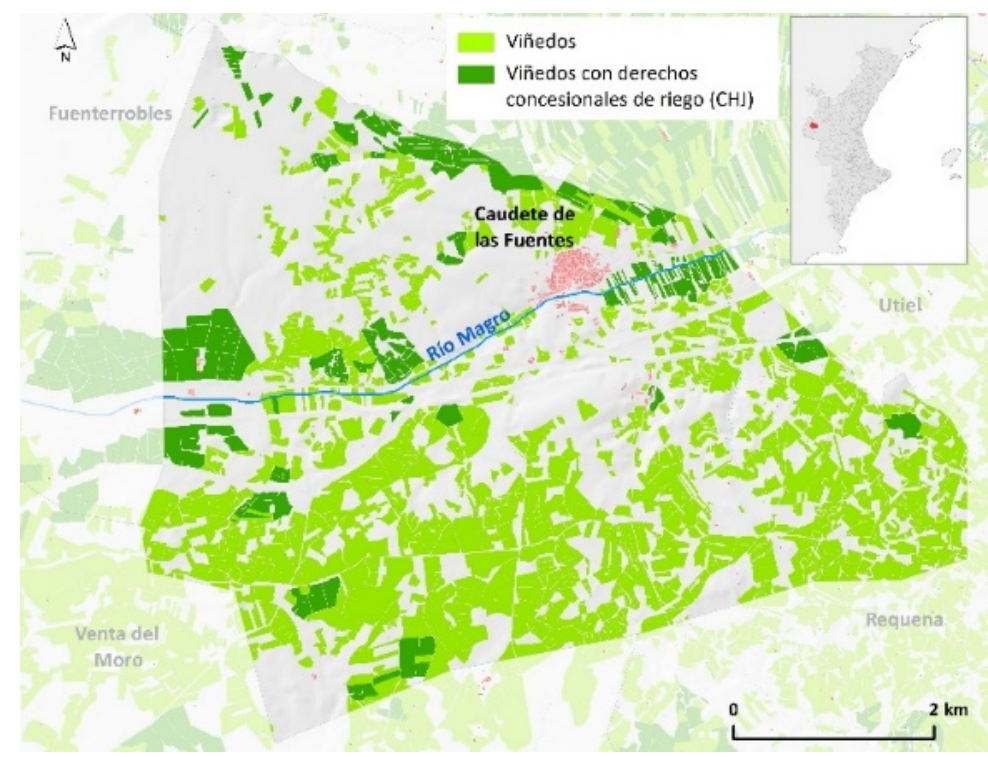

Figura 1. Mapa de localización de la zona de estudio, Caudete de las Fuentes, en la parte central del acuífero Requena-Utiel. Verde claro para viñedos de secano y regadío, y verde oscuro para viñedos que tienen derechos de agua consolidados según la CHJ. 


\section{Congreso Nacional de Riegos CARTAGENA 2021}

\subsection{Información disponible.}

El conjunto de información utilizada para la identificación de las áreas regadas parte de la cartografía oficial del registro público del Sistema de Información Geográfica de Parcelas Agrícolas (SIGPAC). El SIGPAC permite identificar geográficamente las parcelas por tipo de cultivo y aporta información gráfica y alfanumérica de cada una de ellas. A partir de esta información se delimitó geográficamente la superficie total de viñedo del municipio de Caudete de las Fuentes.

\subsubsection{Datos climáticos.}

Los datos de precipitación empleados en el análisis fueron obtenidos del satélite CHIRPS (Climate Hazards Group InfraRed Precipitation with Station data) del Servicio Geológico de los Estados Unidos (USGS) y la Universidad de California, Santa Bárbara (UCSB). CHIRPS incorpora un conjunto de datos de lluvia mensual en imágenes satelitales de resolución de $0.05^{\circ}$ y utiliza datos de estaciones in situ para crear cuadrículas de series temporales de lluvia para el análisis de tendencias y el monitoreo de sequías estacionales [27]. Partiendo del conjunto de datos, se ha filtrado una colección de imágenes mensuales y se han reducido a una sola imagen, donde cada píxel es la suma de las precipitaciones de todas las imágenes del mes. Un total de 18 imágenes fueron procesadas durante los meses de riego establecidos en el estudio.

De forma paralela, los datos diarios de temperatura de la superficie terrestre (LST) fueron tomados del espectrorradiómetro de imágenes de media resolución (MODIS). Los datos son almacenados con una resolución temporal diaria, reduciéndose a una escala mensual. Un total de 92 imágenes fueron procesadas para establecer la temperatura registrada durante los meses de riego.

\subsubsection{Datos topográficos y morfología del cultivo.}

La condición topográfica de las parcelas ha sido determinante en el análisis debido a su influencia en el almacenamiento de agua en el suelo y, por tanto, al crecimiento de la cobertura vegetal. Para ello, se utilizó el Modelo Digital de Elevación (MDE) del Instituto Geográfico Nacional Español (IGNE). A partir del MDE se obtuvo, por procesamiento digital, otros parámetros derivados como la pendiente y orientación de las parcelas. La información procesada fue incorporada al conjunto de datos. De forma adicional, y sabiendo que las unidades geomorfológicas responden de manera diferente en términos de retención de agua en el suelo, se agregó al conjunto de datos las formaciones geomorfológicas derivadas de la cartografía oficial del Plan de Acción Territorial para la prevención del riesgo de inundaciones en la Comunitat Valenciana (PATRICOVA).

Por otro lado, la morfología del cultivo también juega un papel importante en el desarrollo arbóreo de la planta y por tanto en la forma en que el estado hídrico es reflejado por sus hojas. De ese modo, se incluyó en el análisis las diferentes estructuras de plantación del viñedo presentes en la zona, como son sistema en vaso y en espaldera. Adicionalmente, y debido a que las diferentes variedades de vid presentan distintas pautas de crecimiento y requerimientos hídricos, la información varietal se incorporó al conjunto de datos. La variedad bobal es la más frecuente, seguida de garnacha tinta, merlot, messeguera y parellada.
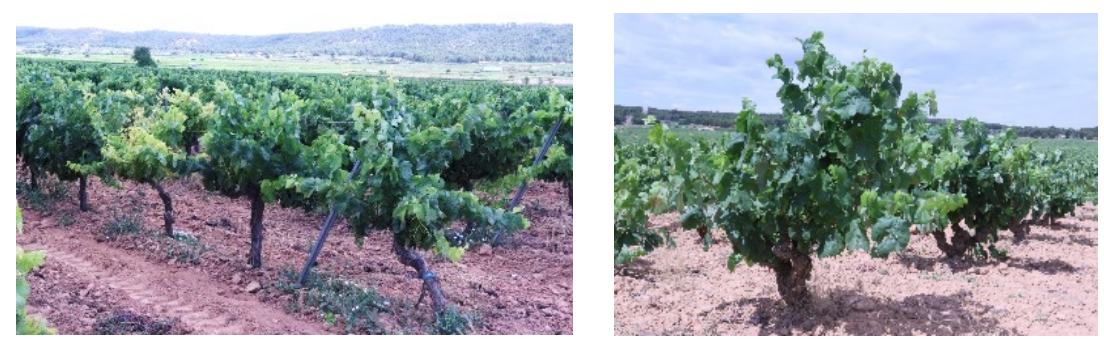

Figure 2. Morfología del cultivo en función de su plantación: sistema en espaldera y sistema en vaso, respectivamente. 


\subsection{Metodología.}

\subsubsection{Método OPTRAM.}

El modelo se basa en la relación física lineal entre la humedad del suelo (W) y la reflectancia del infrarrojo de onda corta transformado (STR) (2) y se parametriza en función de la distribución espacial del píxel entre un índice de vegetación y el STR.

$$
\mathrm{W}=\frac{\theta-\theta_{d}}{\theta_{w}-\theta_{d}}=\frac{S R T-S T R_{d}}{S T R_{w}-S T R_{d}}
$$

Donde STR está relacionado con la banda SWIR de la siguiente manera:

$$
S T R=\frac{\left(1-R_{S W I R}\right)^{2}}{2 * R_{S W I R}}
$$

$Y$ donde STRd y STR $\mathrm{w}$ son STR en seco (por ejemplo, $\theta \sim 0 \mathrm{~cm} 3 \mathrm{~cm}-3$, donde $\theta$ es el contenido de humedad volumétrica) y STR húmedo (por ejemplo, $\theta=\theta \mathrm{s} \mathrm{cm} 3 \mathrm{~cm}-3$, donde $\theta$ s es el contenido de humedad saturada), respectivamente [23].

Investigaciones recientes han mostrado que los índices de vegetación proporcionan una representación de la variabilidad de la vegetación, y esto a su vez está relacionado con el estrés hídrico del suelo [28]. Es decir, SM influye en la cantidad de agua que tiene el cultivo y esto se refleja en las características espectrales de la vegetación. Por lo tanto, se ha empleado el Índice de Vegetación Ajustado de Suelo Modificado (MSAVI2), por su mayor sensibilidad a la vegetación donde el suelo juega un papel significativo en la cobertura [29-31], y que ha sido aplicado en investigaciones recientes [24, 32].

Suponiendo una relación lineal empírica de $S T R_{d}$ y $S T R_{w}$ con la fracción de vegetación, se definen los bordes secos y húmedos del llamado trapezoide óptico como sigue (ver Fig. 3).

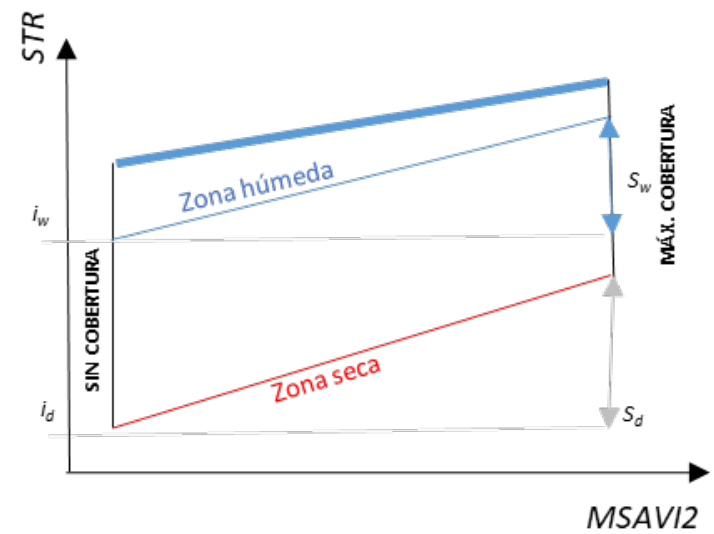

Figura 3. Esquema que ilustra los parámetros del modelo Optico TRapezoide [Eq. (4 y 5) dentro del espacio STR-MSAVI2, (Sadeghi, Jones y Philpot, 2015; Del et al., 2019).

Por lo tanto, STR responde a la relación lineal en el límite húmedo y seco, de la siguiente manera:

$$
\begin{aligned}
& S T R_{d}=i_{d}+s_{d} \text { MSAVI } \\
& S T R_{w}=i_{w}+s_{w} \text { MSAVI }
\end{aligned}
$$

Al combinar las ecuaciones, la humedad del suelo en cada píxel se puede estimar en función de STR y MSAVI2:

$$
W=\frac{i_{d}+s_{d} M S A V I-S T R}{i_{d}-i_{w}+\left(s_{d}-s_{w}\right) M S A V I}
$$




\section{Congreso Nacional de Riegos CARTAGENA 2021

A partir de la interpretación visual, los coeficientes de parametrización entre borde húmedo y seco utilizados para calcular la estimación de humedad normalizada (W), son los siguientes:

$$
\begin{aligned}
& S T R_{d}=0.15+0.7 * M S A V I \\
& S T R_{w}=0.60+0.9 * M S A V I
\end{aligned}
$$

La reflectancia es una función que depende únicamente de las propiedades del suelo y no de las condiciones atmosféricas. Por tanto, suponemos una parametrización universal válida para todas las fechas en un sitio específico [33].

\subsubsection{Random Forest Classifier}

Para la estimación del área regada se utilizó el algoritmo de Random Forest (RF) para construir un modelo clasificador a partir de las variables ambientales obtenidas con métodos ópticos de observación terrestre. RF es un conjunto de algoritmos de aprendizaje automático que consta de una gran cantidad de clasificadores de árboles de decisión, llamados estimadores, donde cada árbol produce sus propias predicciones. La precisión del clasificador de RF depende de la fuerza de los clasificadores de árboles individuales y de la dependencia entre ellos, [34]. Su arquitectura permite manejar grandes conjuntos de datos.

Se aplicó el clasificador de RF utilizando el conjunto de datos del procesamiento de datos ópticos de variables climáticas, topográficas y morfológicas del cultivo, junto a muestras de entrenamiento de un conjunto de datos del estado hídrico del riego en las parcelas del área de estudio.

\section{Resultados y discusión.}

Los valores altos de humedad del suelo en la estación seca son indicadores de la presencia de riego. Sin embargo, empleando únicamente índices de humedad del cultivo no es suficiente para identificar y delimitar las áreas regadas. Parcelas donde la topografía confiere una situación satisfactoria para que se den buenas condiciones hídricas del cultivo, como por ejemplo los barrancos y fondos de valle, hacen difícil clasificar los umbrales entre parcelas regadas y no regadas.

De un total de 2804 parcelas de viñedo, los resultados de la clasificación de suelo regado utilizando la humedad del suelo junto a la topografía, y morfología del cultivo han demostrado mejorar la caracterización de suelo regado con una precisión general del 94.7\%.

La evaluación aporta un error medio cuadrático de 0.163 y R-cuadrado de 0.874 . Los resultados de la evaluación de precisión se llevaron a cabo con una matriz de confusión [35], donde los grupos se asignan a categorías relacionadas con la categoría real probada como datos reales o de referencia. El $75 \%$ de los datos fueron para entrenamiento (227 parcelas) y los datos del $25 \%$ se utilizaron como prueba de validación (76 parcelas).

La precisión se calculó mediante errores de inclusión y exclusión en la clasificación, tabla 1.

Tabla 1. Matriz de confusión

\begin{tabular}{ccc}
\hline & Parcela No Riego & Parcela en Riego \\
\hline Parcela en No Riego & 22 & 1 \\
Parcela en Riego & 1 & 51 \\
\hline
\end{tabular}

El uso de clasificadores que determinan las condiciones hídricas de las plantas por aprendizaje automático proporciona una alta precisión para distinguir las parcelas que han sido regadas en la temporada de riego. La superficie total regada de cultivo de viñedo en el municipio de Caudete de las Fuentes fue de 566.9 ha en el periodo considerado. 


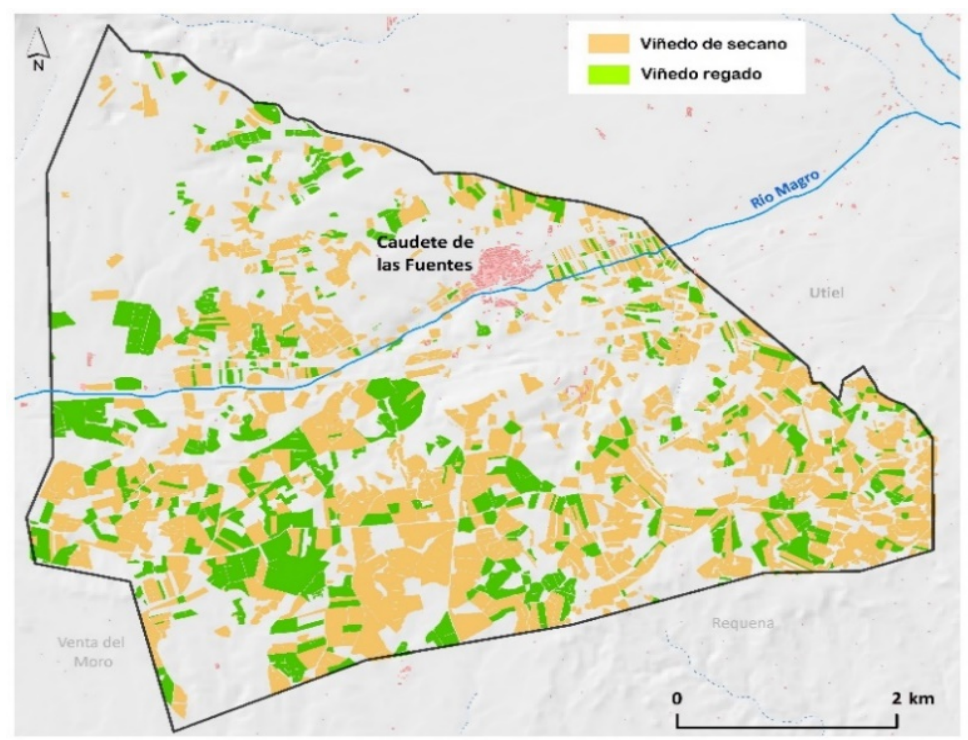

Figura 4. Mapeo de la superficie regada de viñedo en Caudete de las Fuentes para el periodo de riego de 2019.

\section{Conclusiones}

El cálculo de humedad de suelo con imágenes aéreas en el dominio óptico junto a variables que condicionan el estado hídrico del cultivo hace posible distinguir con una precisión de $94.7 \%$ la superficie regada de viñedo. Esto permite evaluar la situación actual y estudiar la evolución de los regadíos aplicando técnicas con sensores remotos.

Se debe tener en cuenta que, cuando se emplean las técnicas de inteligencia artificial, el método evalúa el error del modelo en general sobre el conjunto de datos disponible, pero no sobre cada predicción particular, por lo que el error podría ser mayor si se extrapolan los resultados.

Por tanto, la evaluación del modelo estima un error generalizado en un conjunto de datos limitado y responde a la complejidad real del problema. Para mejorar la estimación se requiere conocer toda la variabilidad existente y predecir así con más exactitud el estado de riego en la parcela.

La alta precisión y los bajos niveles de error obtenidos permiten validar la metodología empleada, que presenta potencial de mejora con una mayor alimentación del proceso de aprendizaje automático. Estas técnicas se aplicarán en breve a otros cultivos leñosos y a toda la masa de agua subterránea de Requena-Utiel.

\section{Agradecimientos.}

Este estudio ha sido financiado por el proyecto eGROUNDWATER (GA No. 1921), que forma parte del programa PRIMA, apoyado por el Programa de Investigación e Innovación Horizon 2020 de la Unión Europea.

\section{Referencias}

1. T. Gleeson, Y. Wada, M. F. P. Bierkens, and L. P. H. Van Beek, 'Water balance of global aquifers revealed by groundwater footprint', Nature, vol. 488, no. 7410, pp. 197-200, 2012, doi: 10.1038/nature11295.

2. D. S. Grogan, D. Wisser, A. Prusevich, R. B. Lammers, and S. Frolking, 'The use and re-use of unsustainable groundwater for irrigation: A global budget', Environ. Res. Lett., vol. 12, no. 3, 2017, doi: 10.1088/1748-9326/aa5fb2.

3. E. López-Gunn, ‘Groundwater governance and social capital’, Geoforum, vol. 43, no. 6, pp. 1140-1151, 2012.

4. T. L. Birkenholtz, 'Recentralizing groundwater governmentality: rendering groundwater and its users visible and governable', WIREs Water, vol. 2, no. 1, pp. 21-30, 2015, doi: 10.1002/wat2.1058. 


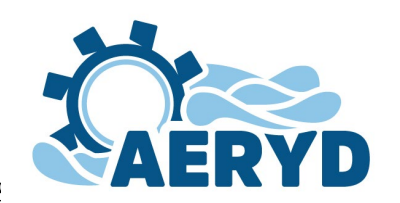

\section{Congreso Nacional de Riegos CARTAGENA 2021}

5. A. Closas, F. Molle, and N. Hernández-Mora, 'Sticks and carrots to manage groundwater over-abstraction in La Mancha, Spain', Agric. Water Manag., vol. 194, pp. 113-124, 2017, doi: 10.1016/j.agwat.2017.08.024.

6. F. Molle, E. López-Gunn, and F. van Steenbergen, 'The local and national politics of groundwater overexploitation', Water Altern., vol. 11, no. 3, pp. 445-457, 2018.

7. F. Molle and A. Closas, 'Why is state-centered groundwater governance largely ineffective? A review', Wiley Interdiscip. Rev. Water, vol. 7, no. 1, pp. 1-17, 2020, doi: 10.1002/wat2.1395.

8. Ostrom, E. (1992): Crafting Institutions for Self-Governing Irrigation Systems, San Francisco: Institute for Contemporary Studies.

9. A. AGRAWAL, 'Common Property Institutions and Sustainable Governance of Resources', WorldDevelopment Vol., vol. 29, no. 10, pp. 1649-1672, 2001, doi: 10.1177/0010836717703674.

10. S. Castaño, D. Sanz, and J. J. Gómez-Alday, 'Methodology for quantifying groundwater abstractions for agriculture via remote sensing and GIS', Water Resour. Manag., vol. 24, no. 4, pp. 795-814, 2010, doi: 10.1007/s11269-009-9473-7.

11. J. F. Brown and M. S. Pervez, 'Merging remote sensing data and national agricultural statistics to model change in irrigated agriculture', Agric. Syst., vol. 127, pp. 28-40, 2014, doi: 10.1016/j.agsy.2014.01.004.

12. D. Sanz, A. Calera, S. Castaño, and J. J. Gómez-Alday, 'Knowledge, participation and transparency in groundwater management', Water Policy, vol. 18, no. 1, pp. 111-125, 2016, doi: 10.2166/wp.2015.024.

13. S. Siebert, V. Henrich, K. Frenken, and J. Burke, 'Update of the digital global map of irrigation areas to version 5 ', Rheinische Friedrich-Wilhelms-Universituy, Bonn, Ger. Food Agric. Organ. United Nations, Rome, Italy, no. August 2014, p. 171, 2013

14. A. K. Ambika, B. Wardlow, and V. Mishra, 'Remotely sensed high resolution irrigated area mapping in India for 2000 to 2015', Sci. Data, vol. 3, pp. 1-14, 2016, doi: 10.1038/sdata.2016.118.

15. J. M. Deines, A. D. Kendall, M. A. Crowley, J. Rapp, J. A. Cardille, and D. W. Hyndman, 'Mapping three decades of annual irrigation across the US High Plains Aquifer using Landsat and Google Earth Engine', Remote Sens. Environ., vol. 233, no. October 2018, p. 111400, 2019, doi: 10.1016/j.rse.2019.111400.

16. M. F. A. Vogels, S. M. de Jong, G. Sterk, and E. A. Addink, 'Mapping irrigated agriculture in complex landscapes using SPOT6 imagery and object-based image analysis - A case study in the Central Rift Valley, Ethiopia -', Int. J. Appl. Earth Obs. Geoinf., vol. 75, no. May 2018, pp. 118-129, 2019, doi: 10.1016/j.jag.2018.07.019.

17. X. Cai, J. Magidi, L. Nhamo, and B. van Koppen, Mapping irrigated areas in the Limpopo Province, South Africa, vol. 172. 2016.

18. A. Calera, I. Campos, A. Osann, G. D'Urso, and M. Menenti, 'Remote sensing for crop water management: From ET modelling to services for the end users', Sensors (Switzerland), vol. 17, no. 5, pp. 1-25, 2017, doi: 10.3390/s17051104.

19. M. Zohaib, H. Kim, and M. Choi, 'Detecting global irrigated areas by using satellite and reanalysis products', Sci. Total Environ., vol. 677, pp. 679-691, 2019, doi: 10.1016/j.scitotenv.2019.04.365.

20. T. N. Carlson and G. P. Petropoulos, 'A new method for estimating of evapotranspiration and surface soil moisture from optical and thermal infrared measurements: the simplified triangle', Int. J. Remote Sens., vol. 40, no. 20, pp. 7716-7729, 2019.

21. T. N. Carlson, 'A brief analysis of the triangle method and a proposal for its operational implementation', Remote Sens., vol. 12, no. 22, pp. 1-9, 2020, doi: 10.3390/rs12223832.

22. M. Sadeghi, S. B. Jones, and W. D. Philpot, 'A linear physically-based model for remote sensing of soil moisture using short wave infrared bands', Remote Sens. Environ., vol. 164, pp. 66-76, 2015, doi: 10.1016/j.rse.2015.04.007.

23. E. Babaeian, M. Sadeghi, T. E. Franz, S. Jones, and M. Tuller, 'Mapping soil moisture with the OPtical TRApezoid Model (OPTRAM) based on long-term MODIS observations', Remote Sens. Environ., vol. 211, no. April, pp. 425-440, 2018.

24. E. Babaeian et al., 'A New Optical Remote Sensing Technique for High-Resolution Mapping of Soil Moisture', Front. Big Data, vol. 2, no. November, pp. 1-6, 2019, doi: 10.3389/fdata.2019.00037.

25. M. Chen et al., 'Evaluation of the OPTRAM Model to Retrieve Soil Moisture in the Sanjiang Plain of Northeast China', Earth Sp. Sci., vol. 7, no. 6, 2020, doi: 10.1029/2020EA001108.

26. C. H. del Júcar, 'PLAN DE EXPLOTACIÓN DE LA MASA DE AGUA SUBTERRÁNEA REQUENA - UTIEL', p. 13, 2016.

27. C. Funk et al., 'The climate hazards infrared precipitation with stations - A new environmental record for monitoring extremes', Sci. Data, vol. 2, no. June 2016, 2015, doi: 10.1038/sdata.2015.66.

28. L. Pádua et al., 'Vineyard variability analysis through UAV-based vigour maps to assess climate change impacts', Agronomy, vol. 9, no. 10, 2019, doi: 10.3390/agronomy9100581.

29. A. J. Richardson and C. L. Wiegand, 'Distinguishing vegetation from soil background information', Photogramm. Eng. Remote Sensing, vol. 43, no. 12, pp. 1541-1552, 1977.

30. J. Qi, A. Chehbouni, A. R. Huete, Y. H. Kerr, and S. Sorooshian, 'A modified soil adjusted vegetation index', Remote Sens. Environ., vol. 48, no. 2, pp. 119-126, 1994, doi: 10.1016/0034-4257(94)90134-1.

31. C. A. Qi J., Kerr Y., 'External_factor_consideration_in_vegetat.pdf'. 1994.

32. P. C. Towers, A. Strever, and C. Poblete-Echeverría, 'Comparison of vegetation indices for leaf area index estimation in vertical shoot positioned vine canopies with and without grenbiule hail-protection netting', Remote Sens., vol. 11, no. 9, 2019, doi: 10.3390/rs11091073. 
33. M. Sadeghi, E. Babaeian, M. Tuller, and S. B. Jones, 'The optical trapezoid model: A novel approach to remote sensing of soil moisture applied to Sentinel-2 and Landsat-8 observations', Remote Sens. Environ., vol. 198, no. September, pp. 52-68, 2017, doi: 10.1016/j.rse.2017.05.041.

34. L. Breiman, 'Random forests', Random For., pp. 1-122, 2001, doi: 10.1201/9780429469275-8.

35. R. G. Congalton, 'A review of assessing the accuracy of classifications of remotely sensed data', Remote Sens. Environ., vol. 37, no. 1, pp. 35-46, 1991, doi: 10.1016/0034-4257(91)90048-B. 